\title{
Radiation field of an ideal thin Gaussian bunch moving in a periodic conducting wire structure
}

\author{
Sergey N. Galyamin* and Viktor V. Vorobev \\ Saint Petersburg State University, 7/9 Universitetskaya naberezhnaya, St. Petersburg 199034, Russia
}

Andrei Benediktovitch

Belarusian State University, 4 Nezavisimosti avenue, Minsk 220030, Belarus

and CFEL, DESY, 85 Notkestrasse, Hamburg 22607, Germany

(Received 15 January 2019; published 26 April 2019)

\begin{abstract}
A theoretical approach for describing the electromagnetic radiation produced by a prolonged electron bunch propagating in the lattice of metallic wires of finite length is presented. This approach is based on the vibrator antenna theory and involves the approximate solving of Hallen's integral equation. For a single wire, it is also supposed that a wire is sufficiently thin and charge motion is relativistic. For many-wire structures, the approximation similar to the kinematic approach of the parametric x-ray radiation theory is additionally applied. The validity of the method is verified by numerical simulations with COMSOL Multiphysics. Possible applications of the interaction between charged particle bunches and artificial wire structures are discussed.
\end{abstract}

DOI: 10.1103/PhysRevAccelBeams.22.043001

\section{INTRODUCTION}

Artificial wire structures that have been attractive to researchers over the past several decades have not lost their importance in recent years. One widespread motivation of mentioned research was the "simulation" of plasma properties for microwave frequencies with the use of a "rodded medium," i.e., the lattice of parallel conducting wires (rods) [1]. Later on, this idea was extensively utilized in the context of development of metamaterials and mainly "left-handed" metamaterials [2]. In the latter case, a "wire medium" was used for providing negative effective dielectric permittivity (within a certain frequency region), while negative effective magnetic permeability in this region was provided by a lattice of "split-ring resonators" [3-5]. Effective electromagnetic (EM) properties of the wire medium itself were also studied in detail [6-8]. For example, the role of spatial dispersion and various possibilities to suppress this effect were discussed [9]. It is worth noting that in the aforementioned investigations the wavelengths under consideration were supposed to be much larger compared to the period of wire structure, therefore allowing an averaged electromagnetic description of the structure with the use of "effective" macroscopic

\footnotetext{
*s.galyamin@spbu.ru

Published by the American Physical Society under the terms of the Creative Commons Attribution 4.0 International license. Further distribution of this work must maintain attribution to the author(s) and the published article's title, journal citation, and DOI.
}

parameters. In this "long-wave" framework, radiation occurring during the passage of a charged particle bunch through a three-dimensional wire medium (i.e., Cherenkov radiation) or in the vicinity of a planar wire structure was actively studied [10-16]. In particular, this long-wave radiation is of essential interest due to its nondivergent properties $[10,11]$.

However, when the bunch is sufficiently short, short wavelengths (comparable with the structure period) can be generated, therefore altering the possibility to describe the wire structure using effective parameters. The portion of the EM radiation related to these short enough wavelengths ("diffraction response" or "short-wave response") can be described using Bragg's diffraction theory formalism, similarly to the parametric x-ray radiation (PXR) in real crystals $[17,18]$. Under the described conditions, the metallic wire assembly can be referred to as a "wire crystal." For example, if the array spacing is of the order of millimeters, the resulting Cherenkov radiation wavelength is in the terahertz $(\mathrm{THz})$ frequency range. Since this range was of significant interest during the past decade due to its prospective applications in various areas [19], corresponding wire structures can be used for the development of efficient $\mathrm{THz}$ radiation sources [20]. It should be noted that the usage of artificial crystals sufficiently changes the connection between the increment of instability and the beam density, resulting in a significant reduction in the beam current needed to reach the stimulated emission effect. This fact allows considering such sources as a new type of free electron laser-volume 
free electron laser [21-23]. Moreover, waveguides loaded with artificial metamaterials (including specific $\mathrm{THz}$ wire crystals) are considered nowadays as prospective candidates for the development of high-power and highgradient accelerators [24,25].

In our previous paper [26], we have started developing the universal approach for the investigation of EM radiation produced by a charged particle bunch moving through the wire structure composed of finite length perfectly conductive wires. In this paper, we describe this approach in detail and prove its applicability by a comparison between analytical results and results of simulations with COMSOL Multiphysics. In particular, we show that the approximation similar to the kinematic approach of PXR is applicable to the wire structure with thin enough conductors and a sparse enough lattice. It should be emphasized that the presented formalism is valid for a monodirectional, monoenergetic bunch only. Effects of emittance and energy spread as well as beam dynamics are not taken into account.

The paper is organized as follows. After the introduction (Sec. I), we formulate the problem for the EM radiation in a wire structure (Sec. II) and consider in detail the EM response of a single wire (Sec. III). Within this section, two approximations are discussed: the case where radiation influence on the surface current distribution is neglected (Sec. III A) and the case with radiation corrections taken into account (Sec. III B). Section IV contains numerical results, a comparison between simulated and analytical results, and a discussion. Section V finishes the paper.

\section{PROBLEM FORMULATION}

Figure 1 shows the geometry of the problem under consideration. A thin Gaussian bunch (carrying a total charge $q$ and having an rms length $\sigma$ ) with the following charge distribution:

$$
\rho(x, y, z, t)=\frac{q \delta(x) \delta(y)}{\sqrt{2 \pi} \sigma} \exp \left(\frac{-(z-v t)^{2}}{2 \sigma^{2}}\right)
$$

traverses with constant velocity $v=\beta c$ the periodic lattice of perfectly electric conductive (PEC) cylinders distributed

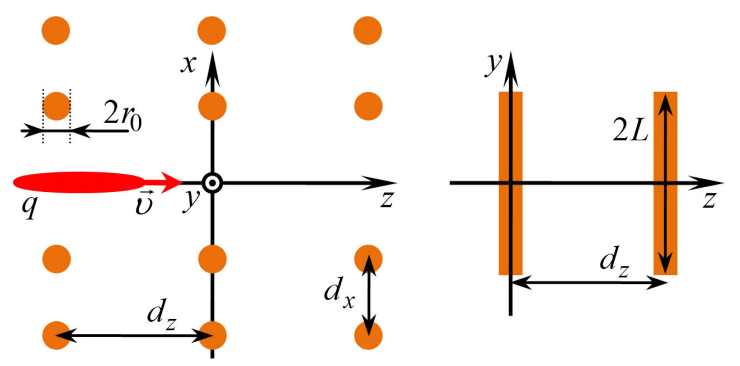

FIG. 1. Geometry of the structure: Perfectly conductive wires of length $2 L$ and radius $r_{0}$ form a rectangular lattice with periods $d_{x}$ and $d_{z}$ which is traversed by a Gaussian bunch (1) of charged particles. There are no wires on bunch trajectory $z=0$. in a vacuum. Since the bunch is considered to be infinitely small in the transverse plane, the approach presented below can be referred to as the "1D theory." The cylinders' length is $2 L$, and the cylinders' radius is $r_{0}$. Wires are located in nodes of a rectangular lattice with periods $d_{x}$ and $d_{z}$ excluding the $z$ axis, along which the bunch moves. Therefore, the position of each cylinder's axis $x_{l m}, z_{l m}$ is given by a pair of integers $(l, m)$, so that

$$
x_{l m}=l d_{x}, \quad z_{l m}=m d_{z} .
$$

Below, we will use the following approximation: we suppose that each wire is excited by a Coulomb field of the moving bunch but does not get affected by the field produced by each neighboring wire. This approximation is very close to the "kinematic approach" of the parametric $\mathrm{x}$-ray radiation of charged particle bunches in real crystals. The validity of this approach will be further verified by numerical simulations in COMSOL (see Sec. IV). The approach used below for the calculation of the response of each wire is related to Hallen's method widely used in the antenna theory. For example, the formulation of a typical Hallen's problem for receiving or transmitting a wire antenna can be found in Ref. [27], where the reference to the seminal Hallen paper is also given. Note that here we generalize this approach to the case of excitation by a charged particle bunch. This approximation allows the consideration of a single-wire excitation independently.

\section{SINGLE-WIRE EXCITATION}

In this section, we will calculate the response generated by a single wire with "coordinates" $(l, m)$ aside which a Gaussian bunch moves. The geometry of this subproblem is shown in Fig. 2.

The problem is solved in the frequency domain; therefore, Fourier harmonic amplitudes are considered, i.e.,

$$
\left\{\vec{E}_{\omega}, \vec{H}_{\omega}\right\}=\frac{1}{2 \pi} \int_{-\infty}^{+\infty} d t\{\vec{E}, \vec{H}\} \exp (i \omega t)
$$

The "incident" field ( $r$ component in the cylindrical frame associated with the Cartesian frame $x, y, z$ ) has the form
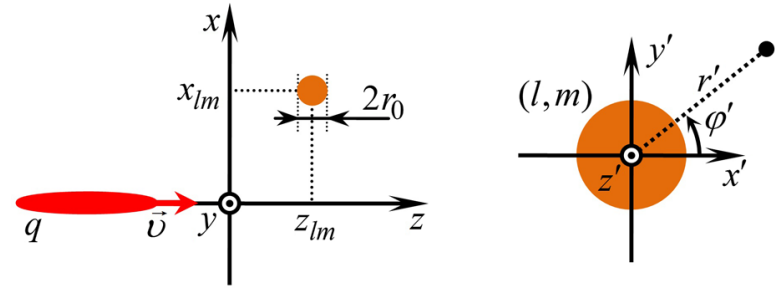

FIG. 2. Single-wire excitation and local coordinate frames (Cartesian frame $x^{\prime}, y^{\prime}, z^{\prime}$ and cylindrical frame $r^{\prime}, \varphi^{\prime}, z^{\prime}$ ) associated with the wire. 


$$
E_{\omega r}^{(i)}=\frac{i q s_{0}}{2 \beta c} H_{1}^{(1)}\left(r s_{0}\right) e^{i(\omega z / v)} e^{-\left(\omega^{2} \sigma^{2} / 2 v^{2}\right)} .
$$

Here $r=\sqrt{x^{2}+y^{2}}, H_{1}^{(1)}$ is the Hankel function, $s_{0}=i \sigma_{0}$, $\sigma_{0}=\sqrt{\omega^{2} v^{-2}\left(1-\beta^{2}\right)}$, and $\operatorname{Re} \sqrt{ }>0$.

For $\sigma=0$, expression (4) corresponds to the field of a point charge moving in a vacuum. Below, the relativistic motion will be considered, $\beta \rightarrow 1$; therefore, $\sigma_{0} \rightarrow 0$. Using asymptotic expressions for the Hankel function [28], the incident field can be simplified, and $E_{r}$ takes the form

$$
E_{\omega r}^{(i)} \approx \frac{q}{\beta \rightarrow 1} \frac{1}{\pi c} \frac{r}{r} \exp \left(i \frac{\omega z}{c}-\frac{\omega^{2}}{\omega_{\sigma}^{2}}\right)
$$

where $\omega_{\sigma}=\sqrt{2} c \beta / \sigma$.

The main problem here is to find the surface current induced at the surface of the wire. We will suppose that wires are thin, i.e.,

$$
r_{0} \ll L
$$

therefore, wire flanges can be neglected. Moreover, we can suppose that the surface current has only a $y$ component and it does not depend on $\varphi^{\prime}$ (see Fig. 2). Therefore, surface current $\vec{j}_{e}^{\text {surf }}$ can be presented as follows:

$$
\vec{j}_{e}^{\text {surf }}=\vec{e}_{y} j_{e}^{\text {surf }}, \quad j_{e}^{\text {surf }}=\frac{I\left(z^{\prime}\right)}{2 \pi r_{0}} \delta\left(r^{\prime}-r_{0}\right),
$$

where $I\left(z^{\prime}\right)$ is the total current of a wire. The current $I\left(z^{\prime}\right)$ should satisfy the boundary conditions at the ends of a wire:

$$
I(L)=I(-L)=0
$$

Vector and scalar potentials satisfy the following equations:

$$
\left(\Delta+k_{0}^{2}\right)\left\{\begin{array}{l}
\vec{A}_{\omega} \\
\Phi_{\omega}
\end{array}\right\}=-4 \pi\left\{\begin{array}{c}
c^{-1} \vec{j}_{e}^{\text {surf }} \\
\rho_{e}^{\text {surf }}
\end{array}\right\}
$$

where $k_{0}=\omega c^{-1}$ and the Lorentz gauge condition is utilized, $\operatorname{div} \vec{A}_{\omega}-i k_{0} \Phi_{\omega}=0$. In accordance with Eq. (7), $\vec{A}_{\omega}=\vec{e}_{y} A_{\omega y}$; therefore, we can conclude from the Lorentz gauge condition that $\Phi_{\omega}=\frac{1}{i k_{0}} \frac{\partial A_{\omega y}}{\partial y}$. For the $y$ component of the electric field, we obtain

$$
E_{\omega y}=-\frac{\partial \Phi_{\omega}}{\partial y}+i k_{0} A_{\omega y}=\frac{i}{k_{0}}\left(\frac{\partial A_{\omega y}}{\partial y^{2}}+k_{0}^{2} A_{\omega y}\right) .
$$

A solution of Eq. (9) for the vector potential is obtained via a convolution between the current and the Green function:

$$
\begin{aligned}
A_{\omega y}\left(r^{\prime}, y\right) & =\frac{1}{c} \iint_{S} j_{e}^{\text {surf }} \frac{\exp \left(i k_{0} R\right)}{R} d S \\
& =\frac{1}{2 \pi c} \int_{-L}^{L} d z^{\prime} I\left(z^{\prime}\right) \int_{-\pi}^{\pi} d \varphi^{\prime} \frac{\exp \left(i k_{0} R\right)}{R},
\end{aligned}
$$

where $S$ is the surface of the cylinder excluding flanges and

$$
R=\sqrt{\left(r^{\prime}\right)^{2}+r_{0}^{2}-2 r^{\prime} r_{0} \cos \varphi^{\prime}+\left(y-z^{\prime}\right)^{2}} .
$$

Let us introduce the "longitudinal potential" $U(y)$ :

$$
U(y)=2 A_{\omega y}\left(r_{0}, y\right),
$$

which is proportional to $A_{\omega y}$ calculated at the surface of the cylinder. After a series of transformations, the connection between $U(y)$ and total current $I(y)$ can be presented as the following integral relation:

$$
U(y)=\frac{2}{c} \int_{-L}^{L} d z^{\prime} I\left(z^{\prime}\right) K\left(y-z^{\prime}\right),
$$

where

$$
\begin{aligned}
K\left(y-z^{\prime}\right) & =\frac{1}{2 \pi} \int_{-\pi}^{\pi} d \varphi^{\prime} \frac{\exp \left(i k_{0} R_{1}\right)}{R_{1}}, \\
R_{1} & =\left.R\right|_{r^{\prime}=r_{0}}=\sqrt{4 r_{0}^{2} \sin ^{2}\left(\varphi^{\prime} / 2\right)+\left(y-z^{\prime}\right)^{2}} .
\end{aligned}
$$

Neglecting the $\varphi^{\prime}$ dependence in the numerator of the integrand's fraction in Eq. (15), we obtain

$$
\begin{aligned}
K\left(y-z^{\prime}\right) & \approx \exp \left(i k_{0}\left|y-z^{\prime}\right|\right) K_{1}\left(y-z^{\prime}\right), \\
K_{1}\left(y-z^{\prime}\right) & =\frac{1}{2 \pi} \int_{-\pi}^{\pi} \frac{d \varphi^{\prime}}{R_{1}},
\end{aligned}
$$

where the kernel function $K_{1}\left(y-z^{\prime}\right)$ can be expressed through the complete elliptic integral of the first kind $K_{e}(\xi)$ [28]:

$$
K_{1}\left(y-z^{\prime}\right)=\frac{1}{\pi a} \varkappa K_{e}(\varkappa)
$$

where $\varkappa=2 r_{0} / \sqrt{\left(y-z^{\prime}\right)^{2}+4 r_{0}^{2}}$. In particular, one can show that $K_{1}\left(y-z^{\prime}\right)$ possesses a logarithmic singularity for $y \rightarrow z^{\prime}$. Besides, $K_{1}\left(y-z^{\prime}\right)=K_{1}\left(z^{\prime}-y\right)$. Finally, we obtain the following integral equation connecting $U$ and $I$ :

$U(y)=\frac{2}{c} \int_{-L}^{L} d z^{\prime} I\left(z^{\prime}\right) \exp \left(i k_{0}\left|y-z^{\prime}\right|\right) K_{1}\left(y-z^{\prime}\right)$.

Now we are able to formulate Hallen's problem for a single passive wire vibrator excited by the Coulomb field of a moving charged particle bunch. The incident field on 
a wire $(l, m)$ has the following form (wire thickness is neglected here):

$\left.E_{\omega y}^{(i)}\right|_{r^{\prime}=r_{0}}=\left.E_{\omega r}^{(i)}\right|_{r^{\prime}=r_{0}} \frac{z^{\prime}}{r_{l m}} \underset{\beta \rightarrow 1}{\approx} \frac{q}{\pi c} \frac{z^{\prime}}{r_{l m}^{2}} \exp \left(i \frac{\omega z_{l m}}{c}-\frac{\omega^{2}}{\omega_{\sigma}^{2}}\right)$,

where $r_{l m}=\sqrt{x_{l m}^{2}+\left(z^{\prime}\right)^{2}}$. The surface current generates the following field at the surface of the wire:

$$
\left.E_{\omega y}\right|_{r^{\prime}=r_{0}}=-\frac{1}{2 i k_{0}}\left(\frac{d^{2} U}{d y^{2}}+k_{0}^{2} U\right) .
$$

The standard boundary condition on the wire surface requires that the total tangential electric field is zero, i.e.,

$$
\left.\left(E_{\omega y}^{(i)}+E_{\omega y}\right)\right|_{r^{\prime}=r_{0}}=0 ;
$$

therefore,

$$
\frac{d^{2} U(y)}{d y^{2}}+k_{0}^{2} U(y)=2 i k_{0} E_{\omega y}^{(i)} .
$$

Ordinary differential equation (22), integral equation (18), and boundary condition (8) formulate Hallen's problem for the surface current $I\left(z^{\prime}\right)$.

Inhomogeneous equation (22) can be solved straightforwardly. The general solution is

$$
U(y)=A_{s} \sin \left(k_{0} y\right)+A_{c} \cos \left(k_{0} y\right)+U_{\mathrm{inh}},
$$

where $A_{s}$ and $A_{c}$ are constants. The particular solution $U_{\text {inh }}(y)$ can be found using the method of variation of constants. Unknown $U_{\text {inh }}(y)$ is decomposed over known solutions $\left[\sin \left(k_{0} y\right)\right.$ and $\left.\cos \left(k_{0} y\right)\right]$ of homogeneous equation (22):

$$
U_{\mathrm{inh}}(y)=B_{s}(y) \sin \left(k_{0} y\right)+B_{c}(y) \cos \left(k_{0} y\right),
$$

where unknown functions $B_{s}(y)$ and $B_{c}(y)$ are found from the system

$$
\begin{aligned}
& \frac{d B_{s}}{d y} \sin \left(k_{0} y\right)+\frac{d B_{c}}{d y} \cos \left(k_{0} y\right)=0, \\
& \frac{d B_{s}}{d y} \cos \left(k_{0} y\right)-\frac{d B_{c}}{d y} \sin \left(k_{0} y\right)=2 i E_{\omega y}^{(i)} .
\end{aligned}
$$

After a series of simple calculations, we obtain

$$
\begin{aligned}
& B_{s}(y)=\frac{2 i q \exp \left(i k_{0} z_{l m}-\omega^{2} / \omega_{\sigma}^{2}\right)}{\pi c}\left[I_{c}(y)-I_{c}(0)\right], \\
& B_{c}(y)=\frac{2 q \exp \left(i k_{0} z_{l m}-\omega^{2} / \omega_{\sigma}^{2}\right)}{c \pi i}\left[I_{s}(y)-I_{s}(0)\right],
\end{aligned}
$$

where

$$
\begin{aligned}
& I_{s}(y)=\int \frac{\sin \left(k_{0} y^{\prime}\right) y^{\prime}}{\left(y^{\prime}\right)^{2}+x_{l m}^{2}} d y^{\prime}, \\
& I_{c}(y)=\int \frac{\cos \left(k_{0} y^{\prime}\right) y^{\prime}}{\left(y^{\prime}\right)^{2}+x_{l m}^{2}} d y^{\prime} .
\end{aligned}
$$

Functions (27) can be expressed through elementary functions, integral sine (si) and integral cosine (Ci) as follows [29]:

$$
\begin{aligned}
2 I_{c}(y)= & \cosh \left(k_{0} x_{l m}\right) \\
& \times\left[\operatorname{Ci}\left(k_{0} y+i k_{0} x_{l m}\right)+\operatorname{Ci}\left(k_{0} y-i k_{0} x_{l m}\right)\right] \\
& +i \sinh \left(k_{0} x_{l m}\right) \\
& \times\left[\operatorname{si}\left(k_{0} y+i k_{0} x_{l m}\right)-\operatorname{si}\left(k_{0} y-i k_{0} x_{l m}\right)\right], \\
2 I_{s}(y)= & \cosh \left(k_{0} x_{l m}\right) \\
& \times\left[\operatorname{si}\left(k_{0} y+i k_{0} x_{l m}\right)+\operatorname{si}\left(k_{0} y-i k_{0} x_{l m}\right)\right] \\
& -i \sinh \left(k_{0} x_{l m}\right) \\
& \times\left[\operatorname{Ci}\left(k_{0} y+i k_{0} x_{l m}\right)-\operatorname{Ci}\left(k_{0} y-i k_{0} x_{l m}\right)\right],
\end{aligned}
$$

where

$\operatorname{si}(y)=-\int_{y}^{\infty} \frac{\sin (\xi) d \xi}{\xi}, \quad \operatorname{Ci}(y)=-\int_{y}^{\infty} \frac{\cos (\xi) d \xi}{\xi}$.

Equations (24), (26), (27), (28), (29), and (30) determine the inhomogeneous solution of Eq. (22). Constants $A_{s}$ and $A_{c}$ in Eq. (23) should be determined from the boundary condition (8). For this, one should solve integral equation (18).

\section{A. Hallen's problem solution in "quasistationary" approximation}

In this section, we describe the simplest approximation (the so-called quasistationary approximation) for a solution of integral equation (18). Let us rewrite (18) equivalently and separate out the "local" term:

$$
\begin{aligned}
U(y)= & \frac{2}{c} \int_{-L}^{L} d z^{\prime} K_{1}\left(y-z^{\prime}\right) \\
& \times\left\{I(y)-\left[I(y)-I\left(z^{\prime}\right) \exp \left(i k_{0}\left|y-z^{\prime}\right|\right)\right]\right\} \\
= & \frac{2}{c} I(y) \int_{-L}^{L} d z^{\prime} K_{1}\left(y-z^{\prime}\right) \\
& -\frac{2}{c} \int_{-L}^{L} d z^{\prime}\left[I(y)-I\left(z^{\prime}\right) \exp \left(i k_{0}\left|y-z^{\prime}\right|\right)\right] K_{1}\left(y-z^{\prime}\right) .
\end{aligned}
$$

Let us denote 


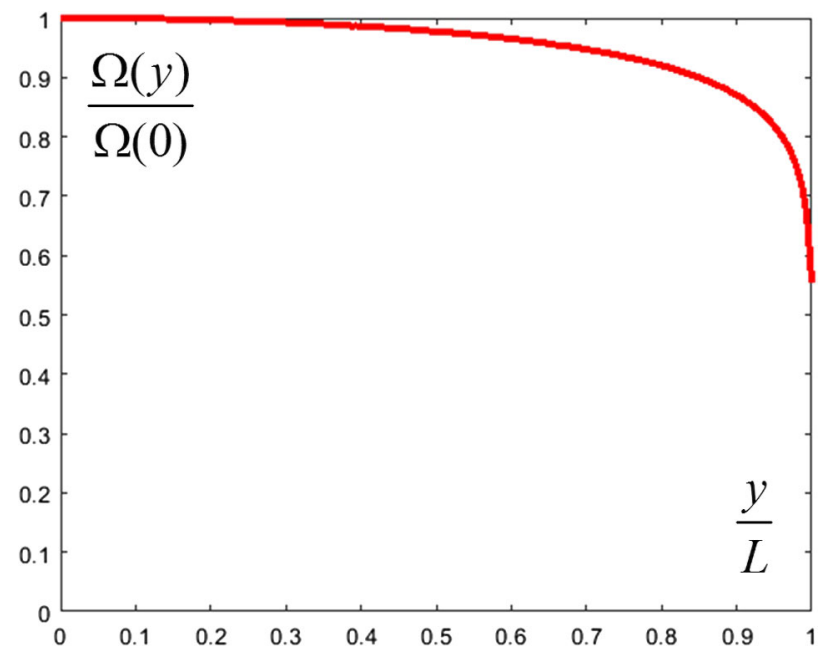

FIG. 3. Typical behavior of $\Omega(y)$ normalized by $\Omega_{0}$ on the halflength of the wire for $L / r_{0}=200$.

$$
\Omega(y)=\int_{-L}^{L} d z^{\prime} K_{1}\left(y-z^{\prime}\right) ;
$$

therefore, $\Omega(-y)=\Omega(y)$. Figure 3 shows the typical behavior of function $\Omega(y)$ for $L / r_{0} \gg 1$. As one can see, this function changes significantly only near $y=L$; therefore, $\Omega(y) \sim \Omega(0)=\Omega_{0}$ for $-L<y<L$. For the calculation of $\Omega_{0}$, the singular kernel $K_{1}\left(y-z^{\prime}\right)$ is frequently substituted by some regular function, for example,

$$
K_{1}\left(y-z^{\prime}\right) \approx \frac{1}{\sqrt{\left(y-z^{\prime}\right)^{2}+r_{0}^{2}}}
$$

therefore, one can obtain an approximate analytical expression, $\Omega_{0} \approx 2 \ln \left(2 L / r_{0}\right)$. The value $\Omega_{0}$ is the large parameter of the problem, in accordance with Eq. (6). However, $\Omega_{0}$ increases just logarithmically (i.e., relatively weakly) with an increase in ratio $L / r_{0}$.

Based on small parameter $\alpha_{0}=1 / \Omega_{0} \sim 1 / \Omega(y)$, one can realize an approximate scheme for solving Eq. (18). Eq. (31) gives

$$
\begin{aligned}
U(y)= & \frac{2}{c} I(y) \Omega(y)-\frac{2}{c} \int_{-L}^{L} d z^{\prime} K_{1}\left(y-z^{\prime}\right) \\
& \times\left[I(y)-I\left(z^{\prime}\right) \exp \left(i k_{0}\left|y-z^{\prime}\right|\right)\right] ;
\end{aligned}
$$

therefore,

$$
\begin{aligned}
I(y)= & \frac{c}{2} \frac{U(y)}{\Omega(y)}+\frac{1}{\Omega(y)} \int_{-L}^{L} d z^{\prime} K_{1}\left(y-z^{\prime}\right) \\
& \times\left[I(y)-I\left(z^{\prime}\right) \exp \left(i k_{0}\left|y-z^{\prime}\right|\right)\right] .
\end{aligned}
$$

The simplest approximation following from Eq. (35) (the so-called quasistationary approximation) consists in neglecting the integral term. In such an approximation, we obtain the local relation between the current $I$ and the longitudinal potential $U$ :

$$
I(y)=\frac{c}{2} \frac{U(y)}{\Omega(y)} .
$$

Boundary condition (8) immediately results in $U( \pm L)=0$; therefore, unknown constants in the general solution (23) can be simply determined. As a result, we obtain

$$
\begin{aligned}
A_{s}= & \frac{2 i q \exp \left(i k_{0} z_{l m}-\omega^{2} / \omega_{\sigma}^{2}\right)}{\pi c \sin \left(k_{0} L\right)} \\
& \times\left[\left(I_{s}(L)-I_{s}(0)\right) \cos \left(k_{0} L\right)-\left(I_{c}(L)-I_{c}(0)\right) \sin \left(k_{0} L\right)\right], \\
A_{c}= & 0 .
\end{aligned}
$$

The fact that $A_{c}=0$ is quite natural, because the incident field on the wire (20) is odd with respect to $z^{\prime}$, and the particular solution of the inhomogeneous equation $U_{\text {inh }}$ is also odd; therefore, the total solution should contain odd functions only. Formulas (36), (23), (24), (26), and (37) solve Hallen's problem for a single wire $(l, m)$ in the approximation described above.

One important peculiarity of this approximate solution is the presence of the term $\sin \left(k_{0} L\right)$ in the denominator of Eq. (37). This term equals zero for resonant frequencies:

$$
\omega= \pm \omega_{m}= \pm 2 \pi f_{m}, \quad f_{m}=\frac{c}{2 L} m, \quad m=1,2, \ldots
$$

For example, the first resonant wavelength $\lambda_{1}=c / f_{1}=$ $2 L$; i.e., it equals the total length of the wire. Therefore, resonances take place when the total wire length is an integer of wavelengths. This resonant condition will be clarified in Sec. III B.

To conclude this subsection, we should note the following. As is known in the antenna theory, the quasistationary approximation described above does not take into account the influence of radiation on the distribution of surface current along the wire. An analog of this approximation from the circuit theory is a passive oscillatory circuit without active resistance (without dissipation) possessing a singularity for a resonant frequency. Taking into account active resistance resolves this singularity. For a passive vibrator, taking into account the dissipation is equivalent to taking into account the radiation and its effect on the current distribution.

\section{B. Hallen's problem solution with radiation taken into account}

First, let us obtain a solution of Eq. (18) with higher accuracy (with respect to small parameter $\alpha_{0}$ ) compared to the local solution (36). To do this, we substitute into the square brackets of the integrand in Eq. (35) the local solution (36). Since the integral in Eq. (35) is already multiplied by small parameter $\alpha_{0}$, such a substitution is sufficient to obtain the first-order correction to the local solution (36). We obtain 


$$
\begin{aligned}
I(y)= & \frac{c}{2} \frac{U(y)}{\Omega(y)}+\frac{c}{2 \Omega(y)} \int_{-L}^{L} d z^{\prime} K_{1}\left(y-z^{\prime}\right) \\
& \times\left[\frac{U(y)}{\Omega(y)}-\frac{U\left(z^{\prime}\right)}{\Omega\left(z^{\prime}\right)} \exp \left(i k_{0}\left|y-z^{\prime}\right|\right)\right] .
\end{aligned}
$$

In accordance with the form of the general solution for $U(y)$, Eq. (23), let us denote

$$
\begin{aligned}
g_{s}(y)= & \frac{c}{2} \frac{\sin \left(k_{0} y\right)}{\Omega(y)}+\frac{c}{2 \Omega(y)} \int_{-L}^{L} d z^{\prime} K_{1}\left(y-z^{\prime}\right) \\
& \times\left[\frac{\sin \left(k_{0} y\right)}{\Omega(y)}-\frac{\sin \left(k_{0} z^{\prime}\right)}{\Omega\left(z^{\prime}\right)} \exp \left(i k_{0}\left|y-z^{\prime}\right|\right)\right], \\
g_{c}(y)= & \frac{c}{2} \frac{\cos \left(k_{0} y\right)}{\Omega(y)}+\frac{c}{2 \Omega(y)} \int_{-L}^{L} d z^{\prime} K_{1}\left(y-z^{\prime}\right) \\
& \times\left[\frac{\cos \left(k_{0} y\right)}{\Omega(y)}-\frac{\cos \left(k_{0} z^{\prime}\right)}{\Omega\left(z^{\prime}\right)} \exp \left(i k_{0}\left|y-z^{\prime}\right|\right)\right], \\
g_{\mathrm{inh}}(y)= & \frac{c}{2} \frac{U_{\mathrm{inh}}(y)}{\Omega(y)}+\frac{c}{2 \Omega(y)} \int_{-L}^{L} d z^{\prime} K_{1}\left(y-z^{\prime}\right) \\
& \times\left[\frac{U_{\mathrm{inh}}(y)}{\Omega(y)}-\frac{U_{\mathrm{inh}}\left(z^{\prime}\right)}{\Omega\left(z^{\prime}\right)} \exp \left(i k_{0}\left|y-z^{\prime}\right|\right)\right] .
\end{aligned}
$$

Taking into account the oddness of the kernel $K_{1}\left(y-z^{\prime}\right)$ and the function $\Omega(y)$, one can show that

$$
g_{s, c}(-y)=\mp g_{s, c}(y), \quad g_{\text {inh }}(-y)=-g_{\text {inh }}(y) .
$$

With these notations, the total surface current is expressed as follows:

$$
I(y)=A_{s} g_{s}(y)+A_{c} g_{c}(y)+g_{\mathrm{inh}}(y)
$$

where $A_{s}$ and $A_{c}$ are unknown constants. Boundary conditions (8) lead to the following inhomogeneous linear system for $A_{s}$ and $A_{c}$ :

$$
\begin{aligned}
A_{s} g_{s}(L)+A_{c} g_{c}(L)+g_{\mathrm{inh}}(L) & =0, \\
A_{s} g_{s}(-L)+A_{c} g_{c}(-L)+g_{\mathrm{inh}}(-L) & =0,
\end{aligned}
$$

with the determinant $D=2 g_{s}(L) g_{c}(L)$. The solution of this system can be easily obtained:

$$
A_{s}=-\frac{g_{\mathrm{inh}}(L)}{g_{s}(L)}, \quad A_{c}=0 .
$$

Again, the fact that $A_{c}=0$ is connected with the oddness of the incident field and the function $U_{\text {inh }}$. Formulas (44), (40), (42), and (46) solve the problem. As one can see from Eqs. (46) and (40), the singularity for resonance frequencies (38) is absent in Eq. (44), since $g_{s}(L) \neq 0$ for $f=f_{m}$. Therefore, the obtained solution is more physical compared to the local solution obtained in Sec. III A.

\section{Scattered electromagnetic field}

Since the total surface current is found, the electromagnetic field scattered by a single wire can be easily calculated. For clarity, let us rewrite here the final expression for the current excited at the wire $(l, m)$, obtained in the quasistationary approximation:

$$
\begin{aligned}
I^{(l m)}\left(z^{\prime}\right)= & \frac{i q \exp \left(i k_{0} z_{l m}-\omega^{2} / \omega_{\sigma}^{2}\right)}{\pi \Omega\left(z^{\prime}\right)}\left\{\frac{\sin \left(k_{0} z^{\prime}\right)}{\sin \left(k_{0} L\right)}\left[\left(I_{s}(L)-I_{s}(0)\right) \cos \left(k_{0} L\right)-\left(I_{c}(L)-I_{c}(0)\right) \sin \left(k_{0} L\right)\right]\right. \\
& \left.+\left(I_{c}\left(z^{\prime}\right)-I_{c}(0)\right) \sin \left(k_{0} z^{\prime}\right)-\left(I_{s}\left(z^{\prime}\right)-I_{s}(0)\right) \cos \left(k_{0} z^{\prime}\right)\right\}
\end{aligned}
$$

As one can see, the Gaussian nature of the bunch is manifested in the induced surface current via the term $\sim \exp \left(-\omega^{2} / \omega_{\sigma}^{2}\right)$. The vector potential of such a current is calculated via formulas analogous to Eq. (11):

$A_{\omega y}^{(l m)}(x, y, z)=\int_{-L}^{L} d z^{\prime} \frac{I^{(l m)}\left(z^{\prime}\right)}{2 \pi c} \int_{-\pi}^{\pi} d \varphi^{\prime} \frac{\exp \left(i k_{0} R_{0 l m}\right)}{R_{l m}}$,

where

$$
R_{l m}=\sqrt{\left(r_{l m}^{\prime}\right)^{2}+r_{0}^{2}-2 r_{0} r_{l m}^{\prime} \cos \varphi^{\prime}+\left(y-z^{\prime}\right)^{2}},
$$

$r_{l m}^{\prime}=\sqrt{\left(z-z_{l m}\right)^{2}+\left(x-x_{l m}\right)^{2}}$, and at the argument of the exponent in Eq. (48) we have neglected retardation at the width of a wire, in accordance with Eq. (6), that is,

$$
\begin{aligned}
R_{0 l m} & =\left.R_{l m}\right|_{r_{0}=0} \\
& =\sqrt{\left(x-x_{l m}\right)^{2}+\left(z-z_{l m}\right)^{2}+\left(y-z^{\prime}\right)^{2}} .
\end{aligned}
$$

Components of the electromagnetic field (which are of the most interest) are calculated via standard formulas analogous to Eq. (10). In particular, we have

$$
\begin{aligned}
E_{\omega x}^{(l m)} & =\frac{i}{k_{0}} \frac{\partial^{2} A_{\omega y}^{(l m)}}{\partial x \partial y} \\
& =\frac{i}{2 \pi k_{0} c} \int_{-L}^{L} d z^{\prime} \int_{-\pi}^{\pi} d \varphi^{\prime} I^{(l m)}\left(z^{\prime}\right) D_{x y}^{(l m)},
\end{aligned}
$$




$$
E_{\omega z}^{(l m)}=\frac{i}{k_{0}} \frac{\partial^{2} A_{\omega y}^{(l m)}}{\partial x \partial z}=\frac{i}{2 \pi k_{0} c} \int_{-L}^{L} d z^{\prime} \int_{-\pi}^{\pi} d \varphi^{\prime} I^{(l m)}\left(z^{\prime}\right) D_{x z}^{(l m)},
$$

where

$$
\begin{aligned}
D_{x y}^{(l m)}= & \frac{\partial^{2}}{\partial x \partial y} \frac{\exp \left(i k_{0} R_{0 l m}\right)}{R_{l m}} \\
= & \left(x-x_{l m}\right) \frac{\exp \left(i k_{0} R_{0 l m}\right)}{R_{l m}} \times\left\{\left(x-x_{l m}\right)\left[\frac{i k_{0}}{R_{0 l m}}-\frac{r_{l m}^{\prime}-r_{0} \cos \varphi^{\prime}}{r_{l m}^{\prime} R_{l m}^{2}}\right]^{2}+\left(y-y^{\prime}\right)\left[\frac{-i k_{0}}{R_{0 l m}^{3}}+2 \frac{r_{l m}^{\prime}-r_{0} \cos \varphi^{\prime}}{r_{l m}^{\prime} R_{l m}^{4}}\right]\right\} \\
D_{x z}^{(l m)}= & \frac{\partial^{2}}{\partial x \partial z} \frac{\exp \left(i k_{0} R_{0 l m}\right)}{R_{l m}} \\
= & \left(x-x_{l m}\right)\left(z-z_{l m}\right) \frac{\exp \left(i k_{0} R_{0 l m}\right)}{R_{l m}} \\
& \times\left\{\left[\frac{i k_{0}}{R_{0 l m}}-\frac{r_{l m}^{\prime}-r_{0} \cos \varphi^{\prime}}{r_{l m}^{\prime} R_{l m}^{2}}\right]^{2}+\left[\frac{-i k_{0}}{R_{0 l m}^{3}}-\left(\frac{1}{\left(r_{l m}^{\prime}\right)^{2} R_{l m}^{2}}-\frac{r_{l m}^{\prime}-r_{0} \cos \varphi^{\prime}}{\left(r_{l m}^{\prime}\right)^{2} R_{l m}^{4}}\left(\frac{R_{l m}^{2}}{r_{l m}^{\prime}}+2 r_{l m}^{\prime}\right)\right)\right]\right\}
\end{aligned}
$$

Let us discuss the applicability of the obtained results for a field calculation in wire structures containing more than one wire. In our approach, it is supposed that each wire antenna is excited by the field of the moving bunch only. However, rigorously speaking, each wire in a wire structure is also excited by fields produced by all other wires. Therefore, the described approach can be used for manywire structures if a response of each wire is small compared to the field of the bunch. This should be true at least for sufficiently thin wires and a sufficiently sparse lattice. The analogous approximation (usually referred to as the "kinematic approach") is widely used for the consideration of PXR produced by charged particle bunches in real crystals. The validity of the approach developed in this paper (which also can be called the kinematic approach for wire structures) will be proved and discussed in Sec. IV.

\section{NUMERICAL RESULTS AND DISCUSSION}

Here we present results of numerical simulations of electromagnetic processes occurring during the interaction of a charged particle bunch or a point charge with singlewire and many-wire structures and compare this numerical result with analytical results obtained above. For simulations, we have used the frequency domain solver of COMSOL Multiphysics RF module. Corresponding simulated results can be directly compared with analytical ones, because the Fourier harmonics of the scattered field is calculated via formulas (51) and (52) of the developed theory. The structure of the model is shown in Fig. 4. The simulation area is a cylinder with a perfectly matched layer (PML) on its back surface. The scattering boundary condition (SBC) was applied at the "flanges" of this cylinder. Such a combination of boundary
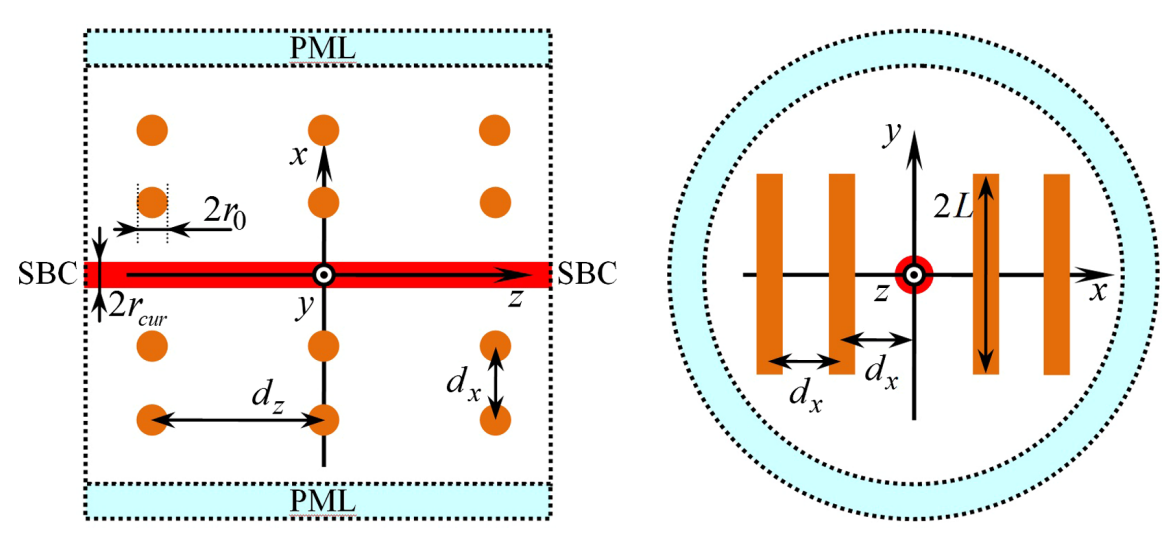

FIG. 4. Simulation area in COMsol Multiphysics. The current cylinder with radius $r_{\text {cur }}$ [inside which the external current density (55) is defined] is shown by red. 
TABLE I. Parameters of simulations in COMSOL Multiphysics.

\begin{tabular}{lc}
\hline \hline Parameter & Value \\
\hline$f(\mathrm{GHz})$ & 10 \\
$\lambda(\mathrm{cm})$ & 3 \\
Length of the simulation area along $z$ & $5 c / \omega \approx 2.4 \mathrm{~cm}$ \\
Radius of the simulation area & $4 c / \omega \approx 1.9 \mathrm{~cm}$ \\
$q(\mathrm{nC})$ & 1 \\
$d_{x}$ & $0.15 c / \omega \approx 0.07 \mathrm{~cm}$ \\
$d_{z}($ short wire $)$ & $0.15 c / \omega \approx 0.07 \mathrm{~cm}$ \\
$2 L$ & $0.2 \lambda=0.6 \mathrm{~cm}$ \\
$2 L($ resonant wire $)$ & $\lambda=3 \mathrm{~cm}$ \\
$L / r_{0}$ & 200 \\
$r_{\text {cur }}$ & $0.01 c / \omega \approx 0.005 \mathrm{~cm}$ \\
\hline \hline
\end{tabular}

conditions allows eliminating the influence of boundaries on the results.

In the frequency domain, a point charge moving along the axis of the structure corresponds to the threadlike current. This current is modeled in COMSOL by a traveling wave of external current density defined inside a cylinder of a small radius $r_{\text {cur }}$ ("current cylinder"). The density of this current has the form

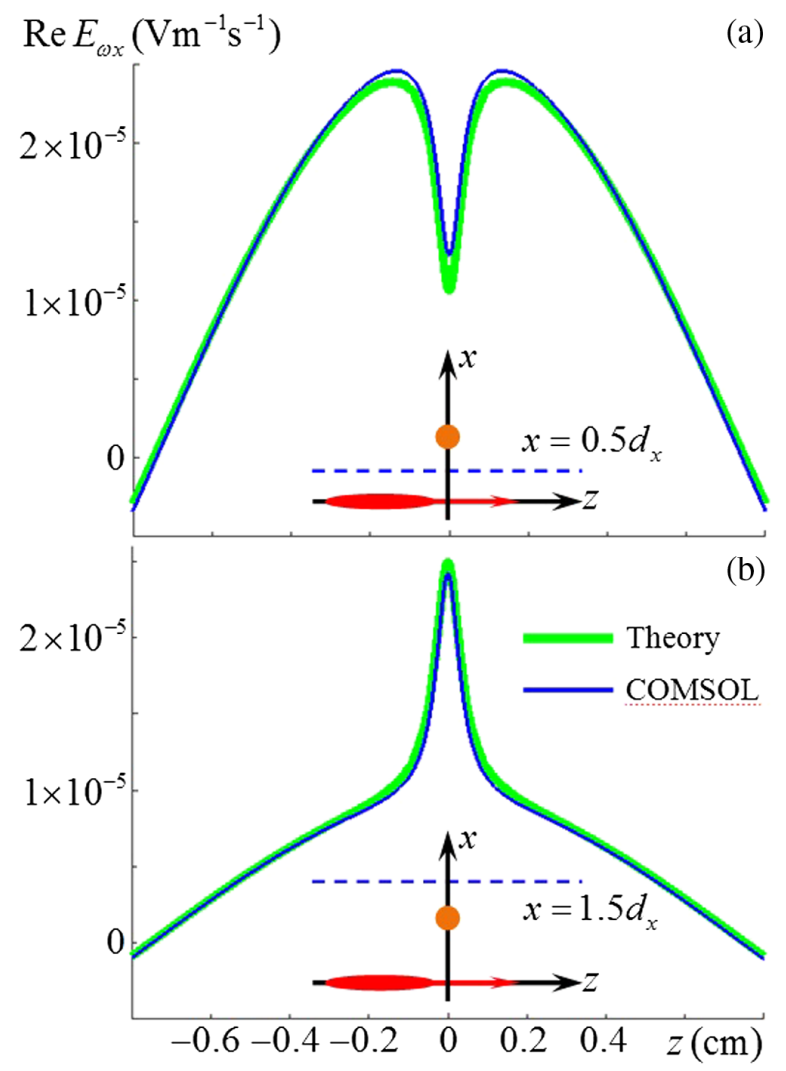

FIG. 5. Real part of $E_{\omega x}$ component over $z$ along the line $x=$ $0.5 d_{x}$ (top) and $x=1.5 d_{x}$ (bottom). A spike for $z=0$ corresponds to the response of a wire. Wire has the "resonant" length, see Table I. $\vec{j}_{\mathrm{ext}}=\vec{e}_{z} I_{\omega 0} \exp \left(i \frac{\omega}{c} z-\frac{\omega^{2}}{\omega_{\sigma}^{2}}\right), \quad I_{\omega 0}=\frac{q}{2 \pi^{2} r_{\mathrm{cur}}^{2}}$

where $r_{\text {cur }} \ll \lambda$ is the radius of the current cylinder and $\lambda=c / f$ is the wavelength under consideration. Current (55) corresponds to the Fourier harmonic of the threadlike current produced by a Gaussian bunch (1) moving with velocity $v=c$. Since the Gaussian nature of the bunch only decreases the magnitude of the current (55), the simulations presented below are performed for $\sigma=0$, i.e., for the case of a point moving charge. It should be noted that the described COMSOL model has been approved in our previous paper [30]. Table I shows the list of parameters used for simulations.

Figure 5 shows a comparison between COMSOL and analytical results for the case of charge flight near a single wire of resonant length (see Table I) with coordinates $(1,0)$.

The real part of the $E_{\omega x}$ component is shown on a line parallel to the $z$ axis for $x=0.5 d_{x}$ [Fig. 5(a)] and for $x=1.5 d_{x}$ [Fig. 5(b)] as a function of $z$. In accordance with Eq. (4), the real part of $E_{\omega x}^{(i)}$ is proportional to $\cos (\omega z / v)$. The background in Fig. 5 corresponds to a half-cycle of this cosine function. An expressed peak for $z=0$ corresponds to the response of a wire, as additionally illustrated by

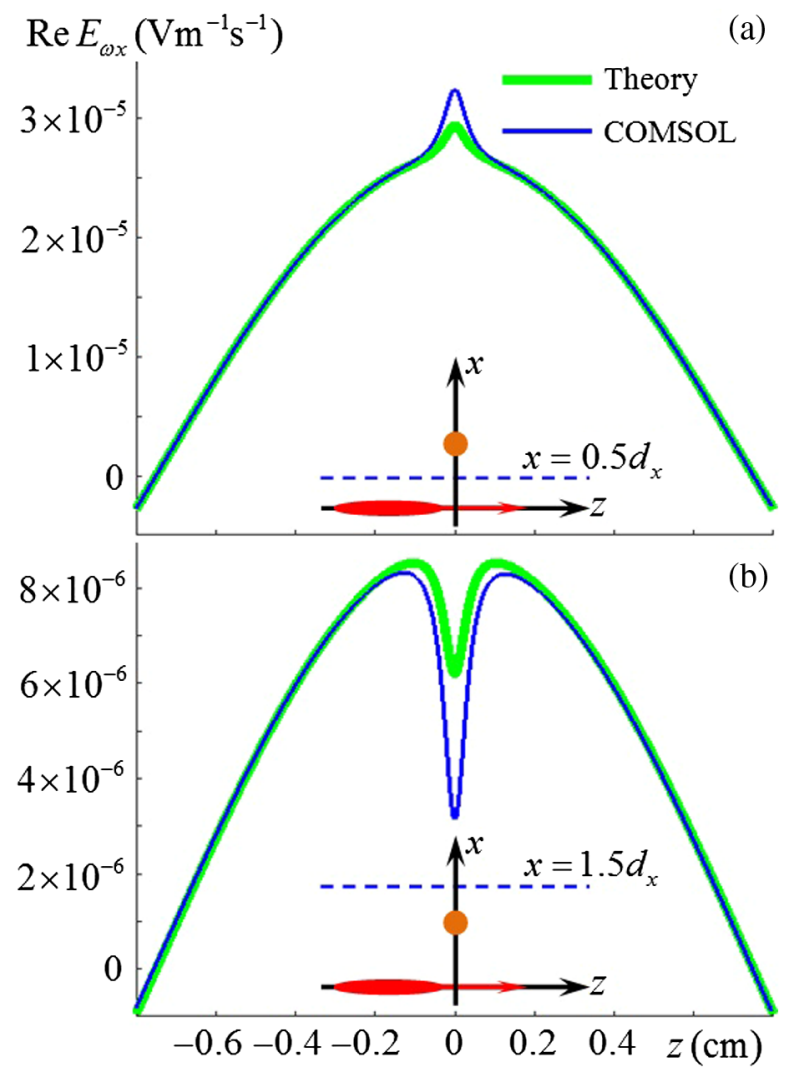

FIG. 6. Real part of $E_{\omega x}$ component over $z$ along the line $x=$ $0.5 d_{x}$ (top) and $x=0.5 d_{x}$ (bottom). A spike for $z=0$ corresponds to the response of a wire. Wire is "short", see Table I. 
insets in Fig. 5. As one can see, the curves are in very good agreement.

Figure 6 shows a comparison between COMSOL and analytical results for the case of a charge flight near a single "short" wire (see Table I) with coordinates $(1,0)$. As one can see, the agreement between curves is worse, and COMSOL gives a stronger response compared to the theory. Moreover, the sign of a spike (which is responsible for the wire response) has a different sign compared to the case of "resonant wire" (see Fig. 5). The magnitudes of spikes are also smaller compared to the resonant wire.

Figure 7 shows the EM field for the case of a charge flight near a string of four short wires with coordinates $(1, m), m=-3,-2,-1,0$. As one can see, the agreement between curves is similar to those in Fig. 6. However, one can conclude from Fig. 7 that each wire gives an independent response. This fact proves the applicability of the kinematic approach for wire structures used throughout this paper (each wire is excited by a Coulomb field of a charge only).

One should mention that for the case of short wires we have observed a bit worse agreement between analytical and COMSOL results compared to the case of resonant wires. A possible reason for this is that the presented analytical consideration is based on an approximate solution of the

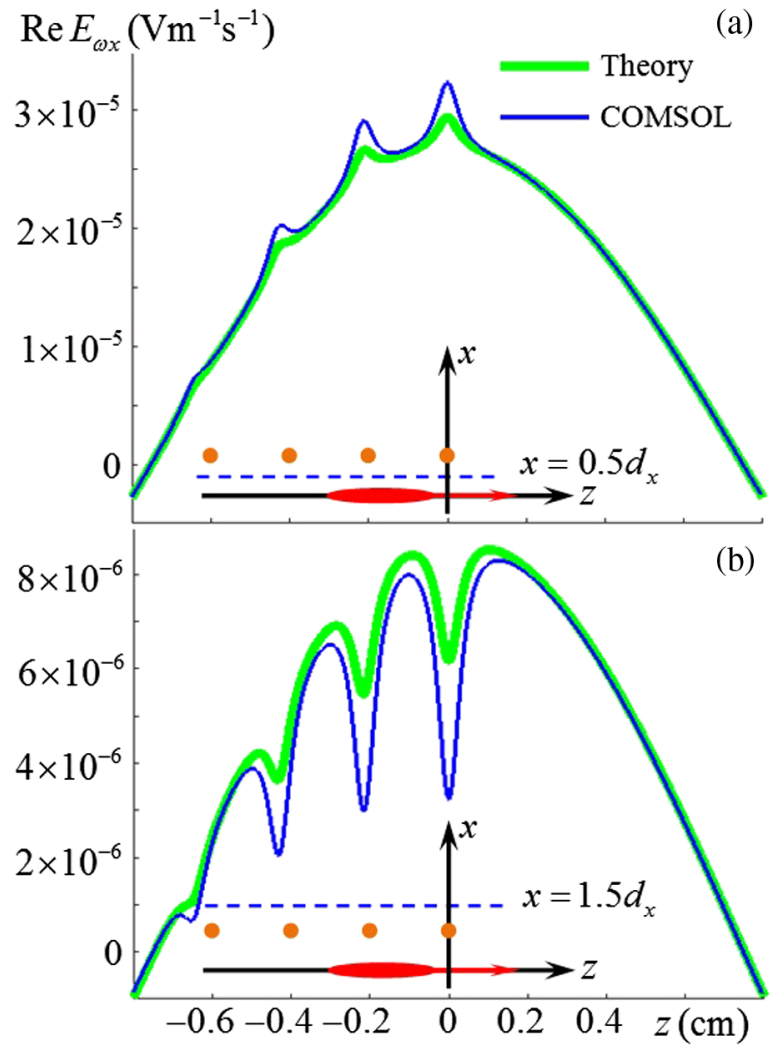

FIG. 7. The real part of the $E_{\omega x}$ component over $z$ along the line $x=0.5 d_{x}$ (top) and $x=1.5 d_{x}$ (bottom) for the case of four short wires with coordinates $(1, m), m=-3,-2,-1,0$. integral equation (18). In the present paper, we used this relatively simple solution to illustrate the analytical method itself. In principle, this issue can be overcome by using numerical procedures for solving the mentioned integral equation with controlled accuracy which can potentially improve the agreement between results. However, the presented approximate solution gives surprisingly good results for a single wire of resonant length. For many-wire structures, more sophisticated schemes taking into account the interaction between wires can be also utilized (see, for example, Ref. [31]). In the context of using wire structures for the generation of EM radiation (in particular, in the $\mathrm{THz}$ frequency range), the resonant case is of most interest, since the surface current magnitude (and, therefore, the radiated EM field) is expected to be much larger in this case compared to the nonresonant wire. For a potential $\mathrm{THz}$ source with $f=1 \mathrm{THz}$, the resonant length of a wire is equal to wavelength $\lambda=0.3 \mathrm{~mm}$. In this case, carbon nanotubes having their radii of the order of nanometers and their lengths from tens of nanometers to several centimeters can be used as thin wire antennas satisfying the inequality (6), and a similar analytical approach can be applied [32]. To generate this frequency, the bunch should be also short enough. For example, for $\sigma \approx 0.03 \mathrm{~mm}$, we have $\omega_{\sigma} \approx 2 \pi \cdot 2 \mathrm{THz}$, and the Gaussian attenuation term $\exp \left(-\omega^{2} / \omega_{\sigma}^{2}\right) \approx 0.78$, which is reasonable.

\section{CONCLUSION}

In the present paper, we have presented an approximate analytical approach enabling the calculation of EM radiation produced by a charged particle bunch during its passage through various structures composed of finite length PEC wires. Contrary to the effective medium approach and Bragg diffraction formalism, this method is free from limitations on the relation between the wavelength under consideration and structure characteristic dimensions (periods and wire length). The method is applicable for relativistic particles and thin enough wires comprising the structure. The limitation on wire thickness is connected with neglecting the effect of wire flanges and the dependence of a surface current on the azimuth angle. Moreover, it is also supposed that interaction between wires can be neglected, which is closely related to the kinematic approach for PXR in real crystals. However, this limitation is not critical and can be overcome. When the wire lattice is close spaced, interaction between neighboring wires can be taken into account by incorporating corresponding terms into the integral equation. Then, an appropriate numerical solution scheme can be utilized (see, for example, Ref. [31]).

The presented numerical results illustrate good agreement between this approach and simulations performed in the RF module of the COMSOL Multiphysics package. In all cases, the theory captures correctly the field behavior. One can conclude from these results that each wire in a 
many-wire structure responds independently to the Coulomb field of the bunch. Moreover, in the resonant case (wire length equal to the wavelength) the agreement between the theory and COMSOL is close to ideal (for a single wire).

\section{ACKNOWLEDGMENTS}

This work was supported by Russian Foundation for Basic Research (RFBR), Grant No. 17-52-04107, and Belarusian Republican Foundation for Fundamental Research (BRFFR), Grant No. F17RM-026. Numerical simulations with COMSOL Multiphysics have been performed at the Computer Center [33] of the Saint Petersburg State University.

[1] W. Rotman, Plasma simulation by artificial dielectrics and parallel-plate media, IRE Trans. Antennas Propag. 10, 82 (1962).

[2] C. M. Soukoulis, J. Zhou, T. Koschny, M. Kafesaki, and E. N. Economou, The science of negative index materials, J. Phys. Condens. Matter 20, 304217 (2008).

[3] J. B. Pendry, A. J. Holden, D. J. Robbins, and W. J. Stewart, Low frequency plasmons in thin-wire structures, J. Phys. Condens. Matter 10, 4785 (1998).

[4] D. R. Smith, W. J. Padilla, D. C. Vier, S. C. Nemat-Nasser, and S. Schultz, Composite Medium with Simultaneously Negative Permeability and Permittivity, Phys. Rev. Lett. 84, 4184 (2000).

[5] D. R. Smith and N. Kroll, Negative Refractive Index in Left-Handed Materials, Phys. Rev. Lett. 85, 2933 (2000).

[6] P. Belov, S. Tretyakov, and A. Viitanen, Dispersion and reflection properties of artificial media formed by regular lattices of ideally conducting wires, J. Electromagn. Waves Appl. 16, 1153 (2002).

[7] A. V. Tyukhtin and E. G. Doilnitsina, Effective permittivity of a metamaterial from coated wires, J. Phys. D 44, 265401 (2011).

[8] S. I. Maslovski and M. G. Silveirinha, Nonlocal permittivity from a quasistatic model for a class of wire media, Phys. Rev. B 80, 245101 (2009).

[9] A. Demetriadou and J.B. Pendry, Taming spatial dispersion in wire metamaterial, J. Phys. Condens. Matter 20, 295222 (2008).

[10] V. V. Vorobev and A. V. Tyukhtin, Nondivergent Cherenkov Radiation in a Wire Metamaterial, Phys. Rev. Lett. 108, 184801 (2012).

[11] D. E. Fernandes, S. I. Maslovski, and M. G. Silveirinha, Cherenkov emission in a nanowire material, Phys. Rev. B 85, 155107 (2012).

[12] A. V. Tyukhtin and V. V. Vorobev, Cherenkov radiation in a metamaterial comprised of coated wires, J. Opt. Soc. Am. B 30, 1524 (2013).

[13] A. V. Tyukhtin and V. V. Vorobev, Radiation of charges moving along the boundary of a wire metamaterial, Phys. Rev. E 89, 013202 (2014).
[14] A. V. Tyukhtin, V. V. Vorobev, and S. N. Galyamin, Radiation excited by a charged-particle bunch on a planar periodic wire structure, Phys. Rev. ST Accel. Beams 17, 122802 (2014).

[15] A. V. Tyukhtin, V. V. Vorobev, and S. N. Galyamin, Radiation of charged-particle bunches passing perpendicularly by the edge of a semi-infinite planar wire structure, Phys. Rev. E 91, 063202 (2015).

[16] V. V. Vorobev, A. V. Tyukhtin, and S. N. Galyamin, Surface waves generated by charged particle bunch moving along the edge of semi-infinite planar wire grid, Radiat. Phys. Chem. 133, 91 (2017).

[17] V. G. Baryshevsky and A. A. Gurinovich, Quasi-Cherenkov parametric radiation from relativistic particles passing through a photonic crystal, Nucl. Instrum. Methods Phys. Res., Sect. B 355, 69 (2015).

[18] V. G. Baryshevsky and E. A. Gurnevich, Cherenkov and parametric (quasi-Cherenkov) radiation produced by a relativistic charged particle moving through a crystal built from metallic wires, Nucl. Instrum. Methods Phys. Res., Sect. B 402, 30 (2017).

[19] G. P. Williams, Filling the THz gap—-high power sources and applications, Rep. Prog. Phys. 69, 301 (2006).

[20] S. V. Anishchenko and V. G. Baryshevsky, Cooperative parametric (quasi-Cherenkov) radiation produced by electron bunches in natural or photonic crystals, Nucl. Instrum. Methods Phys. Res., Sect. B 355, 76 (2015).

[21] V. Baryshevsky, K. Batrakov, A. Gurinovich, I. Ilienko, A. Lobko, V. Moroz, P. Sofronov, and V. Stolyarsky, First lasing of a volume FEL (VFEL) at a wavelength range $\lambda \sim 4-6 \mathrm{~mm}$, Nucl. Instrum. Methods Phys. Res., Sect. A 483, 21 (2002).

[22] V. G. Baryshevsky, K. G. Batrakov, A. A. Gurinovich, I. I. Ilienko, A. S. Lobko, P. V. Molchanov, V. I. Moroz, P. F. Sofronov, and V.I. Stolyarsky, Progress of the volume FEL (VFEL) experiments in millimeter range, Nucl. Instrum. Methods Phys. Res., Sect. A 507, 137 (2003).

[23] V. G. Baryshevsky and E. A. Gurnevich, The electron beam instability in a one-dimensional cylindrical photonic crystal, Nonlin. Phenom. Complex Syst. 15, 155 (2012).

[24] P. D. Hoang, G. Andonian, I. Gadjev, B. Naranjo, Y. Sakai, N. Sudar, O. Williams, M. Fedurin, K. Kusche, C. Swinson, P. Zhang, and J. B. Rosenzweig, Experimental Characterization of Electron-Beam-Driven Wakefield Modes in a Dielectric-Woodpile Cartesian Symmetric Structure, Phys. Rev. Lett. 120, 164801 (2018).

[25] X. Lu, M. A. Shapiro, I. Mastovsky, R. J. Temkin, M. Conde, J. G. Power, J. Shao, E. E. Wisniewski, and C. Jing, Generation of High-Power, Reversed-Cherenkov Wakefield Radiation in a Metamaterial Structure, Phys. Rev. Lett. 122, 014801 (2019).

[26] S. S. Chuchurka, A. I. Benediktovitch, S. N. Galyamin, V. Vorobev, and A. Halavanau, Theoretical modeling of electromagnetic field from electron bunches in periodic wire medium, in Proceedings of the 9th International Particle Accelerator Conference (IPAC2018), Vancouver, BC, Canada, 2018 (JACoW Publishing, Geneva, Switzerland, 2018), pp. 3376-3378. 
[27] C. J. Bouwkamp, Hallén's theory for a straight, perfectly conducting wire, used as a transmitting or receiving aerial, Physica (Utrecht) 9, 609 (1942).

[28] Handbook of Mathematical Functions: with Formulas, Graphs, and Mathematical Tables, edited by $\mathrm{M}$. Abramowitz and I. A. Stegun (Dover, New York, 1972).

[29] A. P. Prudnikov, Y. A. Brychkov, and O. I. Marichev, Elementary functions, Integrals and Series (Gordon and Breach, New York, 1986), Vol. 1.

[30] S. Galyamin, A. Tyukhtin, and V. Vorobev, Focusing the Cherenkov radiation using dielectric concentrator: Simulations and comparison with theory, J. Instrum. 13, C02029 (2018).
[31] V. V. Zalipaev, V. A. Vialov, and S. B. Glybovski, Electromagnetic guided waves on infinite and finite periodic linear arrays of thin metallic wires, in Proceedings of 2017 Progress in Electromagnetics Research Symposium-Spring (PIERS) (IEEE, St. Petersburg, Russia, 2017), pp. 37463753.

[32] G. Y. Slepyan, M. V. Shuba, S. A. Maksimenko, and A. Lakhtakia, Theory of optical scattering by achiral carbon nanotubes and their potential as optical nanoantennas, Phys. Rev. B 73, 195416 (2006).

[33] Computer Center of the St. Petersburg State University, http://www.cc.spbu.ru/en. 